\title{
Presenting the Board of Respiration: Vincent Cottin
}

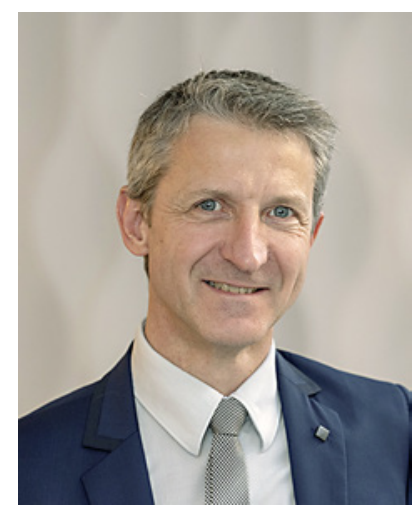

Vincent Cottin

Vincent Cottin is an Associate Editor for Respiration. He works in the field of interstitial lung diseases (ILD) and is a professor in the Department of Pneumology at Hôpital Louis Pradel in Lyon, France.

\section{How Did You Become Interested in ILD?}

I became interested in ILD more than 20 years ago, at a time when ILD and rare lung diseases were still orphan. At that time, there was no treatment for idiopathic pulmonary fibrosis, no treatment for lymphangioleiomyomatosis, etc. The unmet need for patients was huge. I still remember patients from that period whom we were desperate to be helping better.

\section{Which Exciting Developments Are Currently Taking Place in This Field?}

This field has experienced a considerable progress over the last 20 years, with major research developments, two drugs marketed in idiopathic pulmonary fibrosis, and many more still in development. We now know that fibrosis is a very gen- eral process in the body, and that antifibrotic drugs are efficient in any kind of progressive pulmonary fibrosis, which is a major breakthrough that will eventually dramatically alter our management of pulmonary fibrosis in general. Similarly, we have made a lot of progress in the management of connective tissue disease-associated ILD, especially systemic sclerosis, and we now have an effective treatment for lymphangioleiomyomatosis. However, there is still a lot to do and to learn, especially in other rare pulmonary diseases and in pulmonary hypertension associated with ILD.

\section{What Does the Future Hold for the Field, and What Do You Think Are the Main Challenges?}

In the area of ILD and pulmonary fibrosis, we need to learn how to better choose between treatments targeting inflammation and those targeting progressive pulmonary fibrosis. We need to assess whether and how to combine the available therapies. We need biomarkers to monitor progression of fibrosis and activity of inflammation to guide therapy. Also, we now need to tackle the many conditions that have no treatment and for which there is very little research effort at the moment.

\section{What Do You Feel Makes Respiration Special in the Publishing Landscape, and Are There Any Particular Papers You Would Like to See Submitted to the Journal?}

Respiration is unique in that it reflects the longstanding work of a "familial" publisher, independent from the major publishers in medicine. ILD represents only a relatively small proportion of the manuscripts received, but we welcome any good clinical research in ILD! 